\title{
Role of magnetic resonance-high intensity focused ultrasound (MR-HIFU) in uterine fibroids management: an updated systematic review and meta-analysis
}

\author{
Shilin Zheng ${ }^{1}$, Yu Rong ${ }^{2}$, Haiyun Zhü ${ }^{3}$ Xiaoyu Zhang ${ }^{4}$, Xuan Liu ${ }^{1}$, Yun $\mathrm{Wu}^{1}$, Meng Zhao \\ ${ }^{1}$ Department of Interventional Medicine Ward, Wuhan Third Hospital-Tongren Hospital of Wuhan University, Wuhan, China \\ 2Department of Radiology, Guizhou Provincial People's Hospital, Guizhou Provincial Key Laboratory of Intelligent Medical Image Analysis \\ and Precision Diagnosis, Guizhou Provincial People's Hospital, Guiyang, Guizhou, China \\ ${ }^{3}$ Department of Imaging section, Linyi County People's Hospital, Dezhou City, Shandong Province, Shandong Dezhou Linyi, China \\ ${ }^{4}$ Department of Radiology, Affiliated Hospital of Chifeng University, Chifeng, China \\ ${ }^{5}$ Department of Radiology, The First Affiliated Hospital of Nanjing Medical University, Nanjing, China
}

Videosurgery Miniinv 2022; 17 (1): 83-94 DOI: https://doi.org/10.5114/wiitm.2021.109760

\begin{abstract}
Introduction: Magnetic resonance-high intensity focused ultrasound (MR-HIFU) has revolutionized the treatment of Uterine fibroids. Usually, they are associated with prolonged heavy bleeding during the menstrual period, sacral pain, and increased frequency of UTIS, secondary dysmenorrhea, constipation, and pregnancy-associated problems. It also impacts usual activities, which lead to diminished quality of life and rising healthcare costs. Generally, surgery is the only choice for uterine fibroids; however, MR-HIFU is an entirely non-invasive novel therapy, preferred in pregnancy desiring females. Aim: To re-evaluate the efficacy of magnetic resonance-high intensity focused ultrasound (MRHIFU) therapy for uterine fibroids.

Material and methods: Randomized clinical trials (RCTs), prospective or retrospective non-randomized, and crossover studies that considered clinically symptomatic uterine fibroid treatment were included. Meta-analysis was performed using NCSS software, and data were analyzed at a 95\% confidence level with a significance level of 0.05 . In addition, the non-perfused volume percentage (NPV\%), transformed Symptom Severity Score percentage change (tSSS change\%), and health-related quality of life (HR-QoL) were computed.

Results: The overall effect of NPV\% was $67.60 \%$, where the $95 \%$ confidence interval ranged from $55.58 \%$ to $79.62 \%$. The overall impact of $\mathrm{tSSS} \%$ change was approximately 50\% (0.54) with 95\% Cl of 0.41-0.66 of 3 months, 6 months, and 12 months in the included studies. There was a significant improvement in the health-related quality of life (HR-QoL).

Conclusions: The efficacy of MR-HIFU therapy was improved as treatment protocols aimed for total ablation.
\end{abstract}

Key words: uterine fibroids, magnetic resonance-high intensity focused ultrasound (MR-HIFU), health-related quality of life (HR-QoL), meta-analysis, uterine artery embolization (UAE).

\section{Introduction}

Uterine leiomyomas (ULs), also known as myomas or fibroids, are benign smooth-muscle tumors that form in the myometrium and have a low risk of be- coming malignant. They affect around $70 \%$ of women of reproductive age, with Afro-Caribbean women having a 2-3 times greater prevalence, and are the most prevalent reason for gynecologic surgery [1].

\section{Address for correspondence}

Meng Zhao, Department of Radiology, The First Affiliated Hospital of Nanjing Medical University, Nanjing, China,

e-mail: shilinzsl123@sina.com 
Many women are asymptomatic; however, uterine fibroids produce clinically significant symptoms in around $25 \%$ of women [2]. Pelvic discomfort, dysmenorrhea, menorrhagia, urinary frequency, dyspareunia, and subfertility are the most common symptoms. Fibroids may affect fertility, thereby negatively influencing a woman who desires pregnancy. In addition, these uterine fibroids also impact the usual activities, leading to diminished quality of life and increasing healthcare costs [3].

In the formation and expansion of ULS, the amounts of steroid hormones (particularly estrogens and progesterone) and growth factors are critical. Early menstrual periods (under 10 years) have been linked to an increased risk of myomas. It is likely due to the increased number of divisions that myometrial cells undergo during reproductive age, which may predispose to a higher likelihood of mutation in the genes that control myometrial proliferation. Even women with anovulatory cycles marked by increased and extended estrogen production are susceptible to fibroids. ULs may be affected by genetic factors as well. Women with certain genetic disorders such as Alport syndrome, Proteus syndrome, Cowden syndrome, and Reed syndrome, for example, are more likely to acquire ULs [4].

ULs are benign mesenchymal tumors with a spherical form ranging from a few millimeters to several centimeters. Smooth muscle fibers with a concentric spiral pattern and fibrous connective tissue create a pseudo capsule that is attached to the myometrium by fibromuscular bridges. Hyaline, fatty, and cystic fibroids are all potential variations. The accumulation of calcium salts determines calcification. ULs are more frequent in black women than in white women, according to the research [4].

The hydroxylation of the steroid or carbon-2 (2OHE1) or carbon-16 in endogenous estrogen metabolism is predominantly oxidative (16-OHE1). The biological activity is largely controlled by 2-OHE1 metabolites, whereas $16-\mathrm{OHE} 1$ is an estrogen receptor agonist. The CYP1A1 gene appears to be essential for estradiol hydroxylation on carbon 2 since black women with the wild-type CYP1A1 gene had a higher ratio of estradiol derivatives hydroxylated in position 2 than those hydroxylated in position 16 . This viewpoint might explain why black women have a greater rate of ULs [5].

The histological characterization of ULs is a critical element in distinguishing between leiomyoma and leiomyosarcomas. Microscopic inspection and tumor development rate can distinguish UL cells from those of more aggressive tumor types. Atypical or "bizarre" leiomyoma, hemorrhagic cellular leiomyoma, epithelioid leiomyoma, and myxoid leiomyoma are some of the histological types of ULs [4].

Non-random and tumor-specific chromosomal aberrations define ULs, and this points to a connection between these chromosomal changes and tumor physiology. The primary cytogenetic anomalies in ULs were identified by a comparison of many studies, including chromosomal abnormalities t $(12 ; 14)$ (q14-15; q23-24), translocations t $(1 ; 2)$ (p36; p24), and mutations on chromosome 7 (q22-31) [4].

The current understanding of the pathogenic pathways involved in the formation of ULS, particularly the role of biallelic inactivation of fumarate hydratase (FH), mediator complex subunit 12 (MED12), HMGA1, and HMGA2 mutations, was greatly aided by technological advances in high-throughput genomic analysis.

In the pathophysiology of ULS, epigenetic processes play a critical role. Epigenetics is the study of phenotypic changes caused by changes in gene expression. Three major epigenetic processes play a critical role in regulating gene expression in the development of fibroids in humans: microRNAs, DNA methylation, and histone modifications (miRNAs). In tumor samples compared to the myometrium, ULS are linked with changes in DNA methylation at numerous genomic loci and enhanced DNMT1 and DNMT3a mRNA expression [4, 6].

The extracellular matrix of ULS displays immune-reactivity to fibroblast growth factor-1 (FGF) receptor-1, and expression of bFGF-mRNA is higher in ULs than in normal myometrium. In addition, TGF-3 and bFGF are significantly over-expressed in ULS compared to normal myometrium; thus these hormones can help ULs develop faster [4].

Pharmacological treatments are useful in relieving symptoms; however, they may not provide enough control or have substantial adverse effects. Overall, a large majority of patients will require treatment at some point.

Uterine fibroids are still the most common reason for hysterectomy worldwide [3, 7]. For women who wish to start a family, myomectomy is the treatment of choice. On the other hand, surgical procedures are linked with a high risk of short and long-term morbidity, necessitate a hospital stay, and take weeks to recover. Uterine artery embolization (UAE), hysteroscopic resection, and magnetic reso- 
nance-high intensity focused ultrasound are among minimally invasive uterine-sparing therapeutic methods (MRHIFU). MR-HIFU is the only completely non-invasive procedure that has been shown to have numerous benefits, including decreased morbidity, fewer problems, no need for general anesthesia, and a faster recovery period [8, 9].

MR-HIFU is a thermal ablation method that uses targeted tissue heating to treat uterine fibroids non-invasively [10]. The ultrasonic transducer generates high-intensity convergent ultrasound waves. The acoustic energy is absorbed by the targeted tissue, causing a temperature rise and coagulative necrosis and apoptotic cell death [11]. Temperature mapping with magnetic resonance imaging (MRI) improves treatment planning and real-time monitoring [12] Contrast-enhanced MRI can be utilized to visualize the ablated tissue, also known as the non-perfused volume (NPV), immediately after MR-HIFU [12-14].

The NPV percent, which is the NPV divided by the fibroid volume, can describe the treatment outcome [15]. Interference of bowel loops in the beam route during MR-HIFU therapy might result in treatment failure or untreated portions of the fibroid. In order to relocate the bowel loop, several mitigating techniques have been devised. The most frequent approach is the BRB technique i.e. bladder and rectum filling and bladder emptying. There are presently three MR-HIFU devices in clinical use. ExAblate (InSightec, Haifa, Israel) uses the traditional point-by-point ablation approach. The volumetric ablation technology is used in the Sonalleve system (Profound Medical Inc., Toronto, Canada). The Chongqing system (Chongqing Haifu Technology, Chongqing, China) combines shot-sonication with a "point-by-point" therapy method.

The United States Food and Drug Administration (FDA) has authorized MR-HIFU therapy for uterine fibroids since 2004. Due to safety concerns, limited protocols had to be followed at first. However, it became evident over time that the NPV percent is closely connected to treatment results [11, 12]. Fibroids that have been partially ablated tend to regenerate, which might explain the high re-intervention rate reported in trials with a limited procedure $[10,11]$. Furthermore, even when total ablation was sought, MR-HIFU therapy was found to be safe [12].

In 2009, FDA rules were changed to allow operators to strive for full ablation, which has resulted in better results in recent trials $[15,16]$. Furthermore, because uncomplicated pregnancies were recorded after MR-HIFU treatment, the safety recommendations were amended for women with symptomatic uterine fibroids and a desire for future fertility.

Based on the signal intensity of T2-weighted images that differentiated the fibroid composition, Funaki et al. [17] categorized the fibroids into three kinds. Type 1 was a very low-intensity image similar to skeletal muscle; type 2 was an image intensity lower than the myometrium but greater than skeletal muscle; type 3 was an image intensity equal to or higher than the myometrium. However, not all patients are candidates for MR-HIFU therapy. Patient features (BMI and MRI contraindications) or fibroid characteristics evaluated by MR screening might be used as exclusion criteria. Fibroids with a high T2 signal intensity are difficult to treat; therefore, Funaki type 3 fibroids are often avoided [17].

Several studies on the efficacy of MRHIFU therapy for uterine fibroids have been released to date. Overall, they found that MRHIFU successfully reduces symptoms, but there was a high rate of re-intervention [14-17]. However, trials that used restrictive treatment methods that are no longer in use in clinical practice influenced the findings of these analyses.

\section{Aim}

The aim was to re-evaluate the efficacy of MRHIFU in reducing fibroid-related symptoms using treatment protocols that focused solely on total ablation. We also looked at care failures and technical performance as assessed by the post-treatment NPV percent. We also looked at the disease-specific quality of life, re-intervention rates, stability, fertility, costs, and fibroid shrinkage.

\section{Material and methods}

We followed the Preferred Reporting Items for Systematic Reviews and Meta-Analyses (PRISMA) normative recommendations in this study. With the registration number WU \# RC/IRB/2020/1040, the study was sent to the International Prospective Registry of Systematic Reviews (PROSPERO).

\section{Eligibility criteria}

For inclusion, studies on MR-HIFU treatment of women with clinically symptomatic uterine fibroids were reviewed. Treatment procedures that did not target complete ablation (except for a 5-mm pro- 
tection margin from the serosal surface) or ultrasound-guided HIFU systems were ruled out.

RCTs, prospective or retrospective non-randomized, and cross-over studies that considered the treatment of clinically symptomatic uterine fibroids were included. Moreover, patients of more than 18 years of age or at the pre-or peri-menopausal state were also included. Studies that did not have treatment protocols regarded as complete removal of fibroid or used ultrasound-guided HIFU were excluded. Also, animal studies, case reports, and studies not reporting NPV were excluded.

\section{Data search}

The studies were searched in Cochrane Library, PubMed/MEDLINE, SCOPUS, and EMBASE databases. A reference manager (RefWorks) found and deleted duplicate publications, and the articles of the past 5 years were considered, that is, from 2014 to 2019. The first title and abstract screening for all four databases was done independently by two writers (SZ and YR). When research appeared to meet our inclusion criteria, full manuscripts were requested. Other suitable papers for full-text screening were found by manually searching the reference lists of all retrieved full-text publications.

\section{Data extraction}

Data were derived separately from all qualifying studies by the same two authors (SZ and YR). A description of findings table was developed [18-28], which included (a) research characteristics such as authors, year of publication, study style, MRHIFU method, sample size, and follow-up time; (b) treatment parameters: NPV percent, patient eligibility percentage, number of technological errors, use of bowel-interference reduction methods, and sonication duration are all metrics to consider; (c) primary outcome: reduction of fibroid-related symptoms, preferably as measured by the validated disease-specific Uterine Fibroid Symptom and Quality of Life Questionnaire (UFS-QoL); (d) secondary outcomes: health-related quality of life (HRQL), as measured by the UFS-QoL questionnaire, fibroid shrinkage as measured by follow-up MR imaging, and the occurrence of any (serious) adverse events.

The outcomes analyzed were:

- NPV\%, defined by the formula: (non-perfused volume/fibroid volume) $\times 100$ [25-27]
- Fibroid shrinkage is defined as the reduction in the size of the uterine fibroids after MR-HIFU. A higher transformed Symptom Severity Score (TSS) indicates more significant symptom severity [28].

- Health-Related Quality of Life questionnaire concerning uterine fibroid comprises questions asked on a five-point Likert scale, and the score is transformed numerically to a scale from 0 to 100 [29]. A patient who had to undergo a second intervention after MRI-HIFU treatment for uterine fibroid-related problems was considered in the re-intervention percentage.

- Skin burns, menstrual bleeding or unexplained discharge, cystitis, urinary obstruction, constitutional complications, nerve injury, or discomfort for more than 7 days were all considered minor adverse events.

- Patients who needed a second injection due to fibroid-related symptoms were included in the re-intervention percentage (second MR-HIFU, hysterectomy, myomectomy, or UAE).

Disagreements were settled by dialogue or consultation with a third author (MZ). Several reports of a single clinical trial were available, the most recent publication was used as the guide, and additional information was gleaned from secondary journals. We tried to reach the relevant authors by submitting an e-mail with a submission for supplementary data if results were unavailable. A second e-mail was sent if there was no response after 7 days.

\section{Proof quality and the possibility of bias}

Two reviewers separately measured the level of proof in all publications using the Oxford Centre for Evidence-based Medicine (OCEBM) recommendations. Delphi methodology was used to build an 18-criterion method to determine the consistency of the case series. A score of 14 or higher showed high efficiency. Dissension was used to identify and address discrepancies. When the two authors could not agree, a third author was contacted, i.e., MZ.

\section{Data synthesis}

Tables and graphs were used to display the findings of meta-analyses. The change from baseline (\%) was given for continuous data on the same scale (e.g., the difference in fibroid volume). A random-effects model (DerSimonian and Laird) was used to integrate data from relevant studies [26]. If the findings indicat- 
ed statistical heterogeneity $\left(\mathrm{I}_{2}\right)$, we used stratification to explain the disparities. We regarded an $\mathrm{I}_{2}$ value of more than $50 \%$ as indicating significant heterogeneity.

The MR-HIFU device, the usage of bowel-interference mitigation methods, and the length of follow-up were all used to stratify the outcomes.

Meta-analysis was performed using NCSS software. The data were analyzed at a 95\% confidence level with a level of significance of 0.05 . The NPV\%, tSSS change\%, and QoL were computed. Meta-regression was performed to evaluate the association between the different parameters.

\section{Results}

\section{Literature search}

Six hundred eighty-four articles were searched, out of which 454 were excluded because they were either case reports or did not fulfill the research criteria. In addition, 230 articles were screened for the title, out of which 148 were removed because of duplicity. The number of full articles found in the database was 82 , out of which the authors removed 71 articles (Figure 1). The articles included in the meta-analysis numbered 11 . The remaining articles' elimination was based on whether the article used ultrasound-guided HIFU and whether NPV was not evaluated, articles without follow-up, clinical outcome, and restricted treatment as mentioned in $\mathrm{Ta}$ ble I. In addition, articles of the past 5 years were considered, from 2014 to 2019, to re-evaluate the efficacy of MRHIFU therapy for uterine fibroids.

\section{Characteristics of the studies and data extraction}

Morita et al., who reported subjective alleviation of symptoms instead of the tSSS [30], extracted the tSSS from all of the studies included. As a result, this study was omitted from this section of the meta-analysis. Furthermore, because Funaki type 3 patients' tSSS scores could not be assessed as described by Funaki et al., these patients were excluded from our data extraction [18-20, 25]. In addition, uterine fibroids larger than 10 to $12 \mathrm{~cm}$ were also excluded from the analysis [18-20, 23]. Also, Jeong et al. [21] included the effectiveness of MR-HIFU in patients with concomitant adenomyosis. For further information, two writers were contacted. Regrettably, we did not get a response.

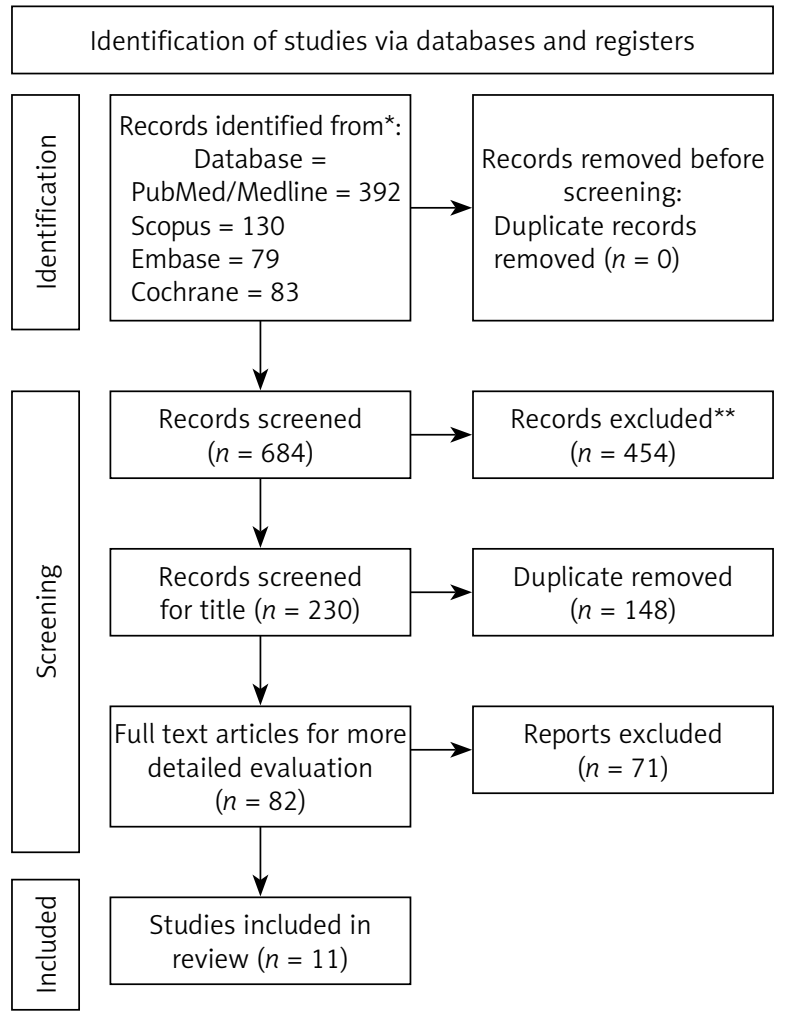

Figure 1. Flow diagram for recruitment and selection of the studies

Table I. Number of articles excluded

\begin{tabular}{|lc|}
\hline Reason for excluding the article & $\begin{array}{c}\text { Number of articles } \\
\text { removed }\end{array}$ \\
\hline Review articles & 22 \\
\hline Ultrasound guided HIFU used & 5 \\
\hline NPV not reported & 12 \\
\hline No follow-up done & 10 \\
\hline Restricted treatment protocol & 15 \\
\hline No clinical outcome & 9 \\
\hline
\end{tabular}

The characteristics of the included studies are tabulated in Table II which describes the type of study or author's name with year of publication, follow-up period considered, outcome measuring NPV, fibroid shrinkage, transformed symptom severity score (tSSS) and health-related quality of life (HR-QoL).

\section{Evidence quality}

Except for one cross-over study, where only the first step was included in our review, all included 
Table II. Characteristics of the studies included

\begin{tabular}{|c|c|c|c|c|}
\hline Study bame & Year & Type of study & Follow-up & Outcome measured \\
\hline Keserci [18] & 2018 & Retrospective & 6 months & $\mathrm{NPV}=97.7 \pm 3.2, \mathrm{FS}=54 \%, \mathrm{tSSS} \%=86 \%, n=72$ \\
\hline Chen [19] & 2016 & Prospective & 6 months & $\begin{array}{c}\mathrm{NPV}=54.8 \pm 21.2, \mathrm{FS}=50.2 \%, \mathrm{tSSS} \%=30.2 \% \\
\text { HR-QoL }=12.9 \%, n=107\end{array}$ \\
\hline Tung [20] & 2016 & Retrospective & 6 months & $\begin{array}{c}\text { NPV }=64.5 \pm 11.4, \text { FS }=31.7 \%, \text { tSSS } \%=43.7 \% \\
\text { HR-QoL }=33.5 \%, n=40\end{array}$ \\
\hline Jeong et al. [21] & 2016 & Retrospective & 3 months & $\mathrm{NPV}=65.6 \pm 22.7, \mathrm{FS}=35.3 \%, \mathrm{tSSS} \%=55 \%, n=157$ \\
\hline Xu et al. [22] & 2015 & Prospective & 6 months & $\mathrm{NPV}=100, \mathrm{FS}=59.1 \%, \mathrm{tSSS} \%=33.5 \%, n=10$ \\
\hline Mindjuk [23] & 2015 & Retrospective & 19.4 months & $\mathrm{NPV}=88.7 \pm 15.0, \mathrm{tSSS} \%=67.8 \%, n=221$ \\
\hline Tan et al. [24] & 2015 & Prospective & 12 months & $\mathrm{NPV}=65.0 \pm 23.0, \mathrm{tSSS} \%=61.8 \%, n=100$ \\
\hline Jacoby [25] & 2014 & Prospective & 3 months & $\begin{array}{c}\mathrm{NPV}=43.0 \pm 20, \mathrm{FS}=18 \%, \mathrm{tSSS} \%=55.4 \% \\
\mathrm{HR}-\mathrm{Q} \mathrm{LL}=56.2 \%, n=13\end{array}$ \\
\hline Park [26] & 2014 & Prospective & 3 months & $\mathrm{NPV}=62.7 \pm 25.5, \mathrm{FS}=27 \%, \mathrm{tSSS} \%=35.6 \%, n=74$ \\
\hline Himabindu [27] & 2014 & Prospective & 6 months & $\mathrm{NPV}=70.0 \pm 20.0, \mathrm{FS}=40 \%, \mathrm{tSSS} \%=59.7 \%, n=32$ \\
\hline Gorny [28] & 2014 & Retrospective & 33.6 months & $N P V=45.5 \pm 22.7, n=138$ \\
\hline
\end{tabular}

FS - Fibroid Shrinkage Percentage, HR-QoL - Health Related Quality of Life.

trials were case series. According to the OCEBM standards of data, all of the included studies had a degree of proof of IV. Using the 18-criteria tool, the consistency of the proof varied from 9 to 16 points, suggesting significant variation in quality between the included studies. The articles included in meta-synthesis numbered 11 . Furthermore, the included studies did not adequately disclose the various statistical parameters, necessitating the estimation of standard deviations. However, where experiments with imputed standard deviations for all result parameters were excluded, predictions for standard deviation imputation were found to be sufficiently robust.

\section{Technical parameters}

\section{Time spent in treatment}

Eleven studies recorded a mean sonication time of $146.2 \mathrm{~min}$. The sonication that took the least amount of time was the method used by Chongqing [18]. The latest findings have shown that extended patient cohorts have shorter waiting periods and that the total treatment period has decreased [22-25].

Overall, the point estimate for overall NPV\% was $67.60 \%$ with a $95 \%$ confidence interval $(\mathrm{Cl})$ of $55.58-79.62 \%, l^{2}$ of $99 \%$, and a $p$-value of 0.00001 , showing statistical significance. The ${ }^{2}$ indicates the amount of variability due to heterogeneity, which could not be explained by stratification or meta-regression. The statistically significant $p$-value indicates that the use of bowel interference mitigation strategies resulted in higher NPV\% (Figure 2).

\section{Symptom evaluation}

Figure 3 shows the overall effect of tSSS\% change as 0.54 with $95 \% \mathrm{Cl}$ of $0.41-0.66$ after a follow-up of 3 months, 6 months, and 12 months. An $r^{2}$ of $93 \%$ showed a significant heterogeneity $p$-value of 0.00001 , showing high statistical significance. The forest plot shows that the diamond shape does not touch the line of no effect, and this means that the symptoms gradually improved after MR-HIFU treatment. The combined estimate of the change percentage for symptoms improvement was 54\%, showing symptom reduction following MR-HIFU treatment. The meta-regression showed no association between NPV\%, fibroid shrinkage, and tSSS\% change (Figure 3).

\section{Health-Related Quality of Life (HR-QoL)}

There were only three studies that evaluated the health-related quality of life. The increase in the tSSS\% indicates a better quality of life following MRHIFU treatment. The quality of life improved after the follow-up, and the combined effect showed an odds ratio of 39.27 with $95 \% \mathrm{Cl}$ of $9.67-87.8$. The studies 


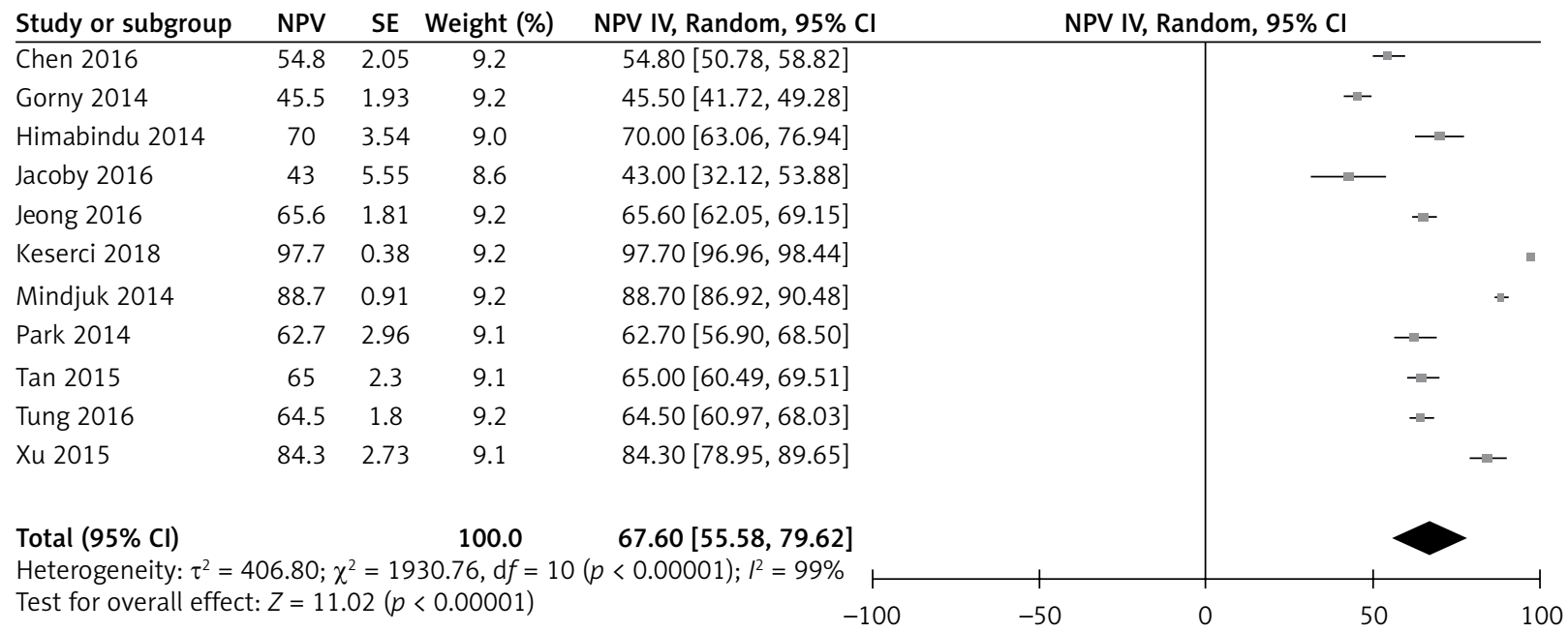

Figure 2. Forest plot of studies included for NPV\%

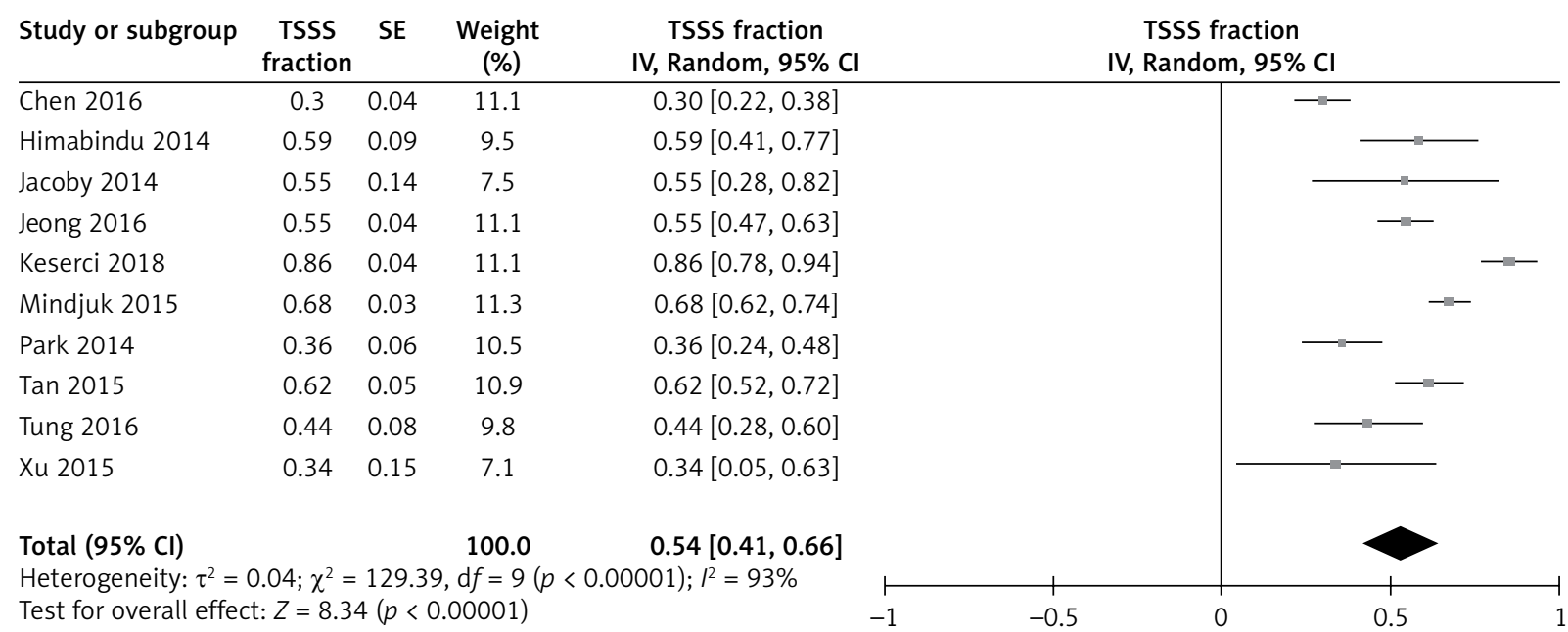

Figure 3. Forest plot of studies included for tSSS change\%

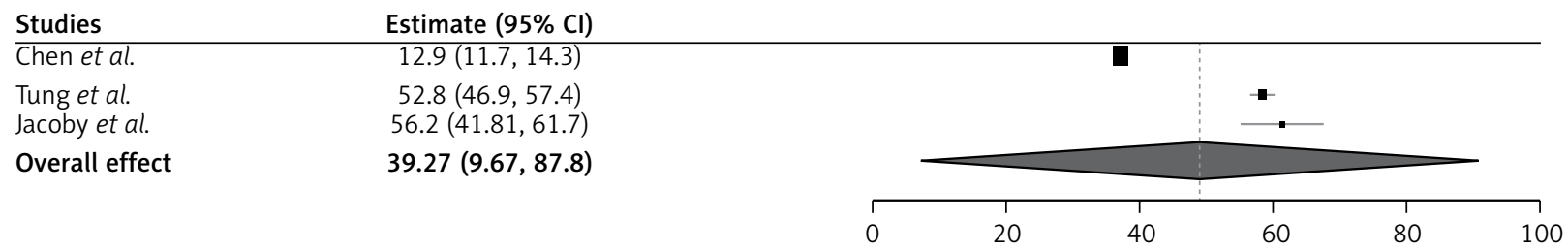

Figure 4. Forest plot of studies included for disease-specific quality of life

have used different devices such as Sonalleve and ExAblate for the MRI-HIFU. The studies that used the Sonalleve device have more adverse effects than the ExAblate device. However, the studies reported not many serious adverse events, which means a better quality of life (Figure 4).

\section{Fibroid shrinkage}

Figure 5 shows fibroid shrinkage after MR-HIFU treatment with a point estimate of $45 \%$ along with $95 \% \mathrm{Cl}$ of $35.50-54.50, p^{2}$ of $96 \%$ and a $p$-value of 0.00001 . The fibroid shrinkage showed significant results in the studies included for review [18, 30, 


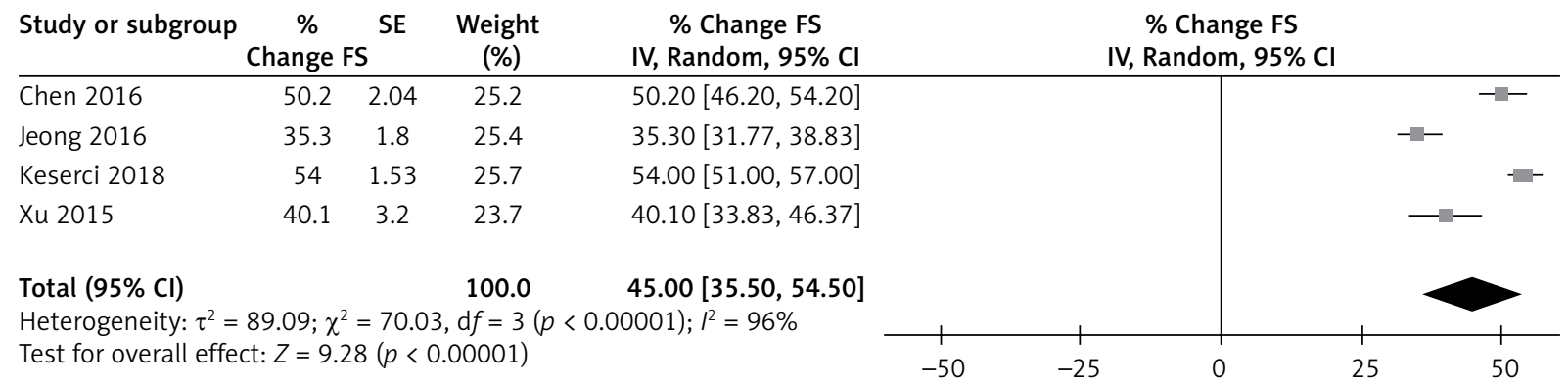

Figure 5. Forest plot of studies included for change in the fibroid shrinkage

31]. After MR-HIFU therapy, all studies demonstrated average fibroid shrinkage. Only minor variations were seen when stratified by follow-up type. Three studies, however, found a significant impact of time on fibroid shrinkage percentage. NPV percent was not statistically correlated with fibroid shrinkage $(p=0.012)$ in an exploratory meta-regression study. However, at the 6-month follow-up, there was a slight difference/trend, indicating a favorable relationship.

\section{Costs}

The writers did not mention any cost-related results. As a result, conclusions about cost-effectiveness cannot be drawn based on the studies used.

\section{Adverse events}

Just one of the studies included in this review did not use $A E$ as an endpoint parameter [13]. One hundred and twenty-four of 1360 patients (9.1\%) had an adverse reaction to one of the 1360 therapies studied. During the follow-up period, 120 AEs were minor and self-limiting. Just $2(0.3 \%)$ patients had a severe adverse effect (SAE), one of which was a deep venous thrombosis (DVT), and the other was a third-degree skin burn. Two of the most recent studies listed these SAEs (patient enrollment between 2005 and 2009) [24, 32]. Sonalleve and ExAblate had a significant gap of (S) AE stratification, $18.4 \%$ and $6.7 \%$, respectively. Meta-regression verified that the difference between Sonalleve and ExAblate was statistically significant $(p<0.05)$. None of the other covariates studied (NPV percent, sonication time) was linked to adverse outcomes. However, not many serious adverse events were reported in the study.

However, a follow-up MRI is expensive and not required. Similarly, re-interventions were found in two studies that showed reintervention needed in $18-24 \%$ of patients.

\section{Discussion}

The present meta-analysis aimed to evaluate the effectiveness of MRI-HIFU in patients with uterine fibroids. The results showed an overall decrease in NPV\% and $\mathrm{tSSS} \%$, and fibroid shrinkage was also evident post-MR-HIFU therapy. The health-related quality of life among patients with uterine fibroids also improved with follow-up; however, this was seen only in three studies and needs to be assessed further. Studies involved in the meta-analysis have also proclaimed improved reproductive outcomes. It has come to light that reintervention was needed in $18-24 \%$ of patients. This meta-analysis included studies that focused on complete ablation to look at the overall effect of MR-HIFU.

The overall level of data, which was low to moderate, affected all of the outcome criteria examined in this study. For inclusion, only non-randomized, non-comparative trials were available. The sources of a high risk of prejudice are linked to the sample designs themselves: insufficient documentation of loss of follow-up and the possibility of selection bias.

Increased expertise improves care effectiveness by reducing technical errors and treatment time in extended patient cohorts. Xu et al. [22] registered the shortest sonication duration, suggesting that the Chongqing method could increase treatment effectiveness. The pooled NPV percent immediately after MR-HIFU was $67.60 \%$, owing to the lack of stringent treatment protocols. The distribution of dispersed points into two classes showed a remarkable asymmetry in our findings. Unfortunately, we were unable to provide a complete explanation. Only a minor disparity was discovered through bowel-in- 
terference avoidance methods, meaning that this may lead to a higher NPV percent.

The pooled tSSS declined on average to 54\% during the follow-up period after MR-HIFU treatment. However, no data were available for more than a year, and MR-HIFU was not linked to other therapeutic choices in any of the trials included. At a 3-month follow-up, Jacoby et al. compared magnetic resonance imaging-guided high intensity focused ultrasound (MRgFUS) to placebo [25] and found that the MRgFUS community had a more significant tSSS decline, - 31 vs. -13 points. We looked for other uterine fibroid studies that used the UFS-QoL questionnaire to compare the tSSS of MR-HIFU to other treatment alternatives (UAE, hysterectomy, and myomectomy). Similarly, studies by Spies et al. [33, 34] and Manyonda et al. [35] also reported a decrease in $\mathrm{tSSS} \%$ change in myomectomy and hysterectomy, and the present meta-analysis indicated an overall tSSS\% change of $54 \%$, which is comparable to hysterectomy and myomectomy.

A very few studies reported the improved health-related quality of life after MR-HIFU treatment; however, the studies included in the present meta-analysis have shown improved uterine fibroid-related quality of life after the treatment.

Fibroid shrinkage was observed in all the studies, and the percentage of shrinkage ranged over time, indicating that fibroids will continue to shrink in volume for at least a year. In the present meta-analysis, the overall fibroid shrinkage after MR-HIFU treatment was around $45 \%$ over a year, showing a statistically significant result $(p<0.05)$. Furthermore, the relationship between fibroid shrinkage and NPV percent was marginally significant, implying that a higher NPV percent could lead to more fibroid shrinkage. Also, it is essential to keep in mind that doing a follow-up MRI exam is not a cost-effective and primarily needless option.

The reintervention reported by Tan et al. [24], Mindjuk et al. [23], and Chen et al. [19] was 9\%, $12.7 \%$, and $0.9 \%$, respectively, while other studies did not report the need for reintervention in the follow-up period. This particular result pointed to the efficacy of the procedure. The procedure of MR-HIFU is non-invasive and does not indicate any effect on the reproductive outcome of the patients after the treatment. None of the studies has included the reproductive outcome and hence raised concern. However, studies by Lee et al. [36] and Cheung et al.
[37] have shown no effect on the anti-Mullerian hormone, indicating that women can try for pregnancy after treating uterine fibroids.

Just two SAEs were identified in older studies [27, 38], which may be clarified by a slight learning curve effect when MR-HIFU was first used in clinical practice [16]. As AE was stratified by method, trials using the Sonalleve system had slightly more $A E$ than trials using the ExAblate device [17, 18, 20, 25]. Two Ex-Ablate reports, on the other hand, reported 'no unforeseen or major AE,' implying under-reporting $[19,38]$. Furthermore, there is no agreement about how to define AE in the context of MR-HIFU. For example, although irregular vaginal discharge is often classified as AE, fibroid expulsion was identified as a common finding in $21 \%$ of ExAblate patients [22]. Surprisingly, a Sonalleve study classified constitutional symptoms as $A E$, although none of the other studies did [25]. Although a reporting bias may clarify the disparity in AE between Sonalleve and ExAblate, more research is needed in the future.

These treatment modalities may have proved to be cost effective [31, 32, 39-41] but were not included in this analysis. Therefore, MR-HIFU can be considered a cost-effective treatment for patients who are ready to pay.

Methodological flaws were to blame for the limitations of the present meta-analysis. Standard deviations were often estimated. There was a lack of follow-up in some trials, and some sub-studies had different sample sizes [42]. As a consequence, the findings should be viewed with caution. Furthermore, since the findings are based on published means rather than actual patient records, the ecological fallacy may have influenced the results. Finally, we questioned whether we should generalize our findings because of the significant and often mysterious heterogeneity in each outcome parameter. However, since we used a random-effects model for meta-analysis [43], this approach is accurate.

Even though MR-HIFU has been used to treat uterine fibroids for 14 years, it is still not widely used or reimbursed worldwide. The gold standard for obtaining reimbursement is a randomized clinical study, and one is currently underway to compare UAE and MR-HIFU [44]. However, they had difficulty selecting volunteers, and some patients refused to be randomly assigned. As a result, randomized studies are challenging to perform and face statistical difficulties. More extensive retrospective random- 
ized cohort trials with longer follow-up are needed to establish the role of MR-HIFU in the management of symptomatic uterine fibroids before they can be used in routine clinical care.

Another challenge associated with uterine fibroids is a misdiagnosis of uterine sarcomas as uterine fibroids because both are evident as focal masses in the uterine myometrium. This is due to non-specific clinical manifestations of uterine sarcomas; they may resemble uterine fibroids or myomas. Uterine sarcomas are rare malignant tumors of the female reproductive system derived from uterine smooth muscle, endometrial stroma, and connective tissue, accounting for $2 \%$ to $6 \%$ of all malignant uterine tumors [45]. MR-HIFU is a new non-invasive thermotherapy with the advantages of high repeatability, uniform thermal diffusion, and conformal treatment based on the actual size and shape of the tumor. It can also monitor temperature changes in the target area and surrounding tissue in real time to adjust ultrasonic power and energy irradiation [46]. Leiomyosarcomas are not sensitive to radiotherapy. However, tumor cells can become sensitive to high temperature [47], resulting in potential complications by increasing the blood flow to the target tissue and enhancing the permeability of the tumor cell membrane via its thermal effects. As there have been no previously reported cases of MR-HIFU alone used to treat uterine leiomyosarcoma [45], it is difficult to identify its role in the misdiagnosis of uterine sarcomas as fibroids.

\section{Conclusions}

The present meta-analysis has shown that it is an entirely effective non-invasive treatment for uterine fibroids; this is evident by reducing the overall NPV, tSSS\% change, and decrease in the fibroid size. Studies focusing on the impact of HR-QoL are needed in this aspect. Furthermore, the trials focusing on the reproductive outcomes should focus on the recommendation of this therapy for uterine fibroids.

\section{Acknowledgments}

Shilin Zheng and Yu Rong both are the first authors, they contributed equally.

\section{Conflict of interest}

The authors declare no conflict of interest.

\section{References}

1. Stewart EA. Uterine fibroids. Lancet 2001; 357: 293-8.

2. Baird DD, Dunson DB, Hill MC, et al. High cumulative incidence of uterine leiomyoma in black and white women: ultrasound evidence. Obstet Gynecol 2003; 188: 100-7.

3. Peitsidis P, Koukoulomati A. Tranexamic acid for the management of uterine fibroid tumors: a systematic review of the current evidence. World J Clin Cases 2014; 2: 893-8.

4. Laganà AS, Vergara D, Favilli A, et al. Epigenetic and genetic landscape of uterine leiomyomas: a current view over a common gynecological disease. Arch Gynecol Obstet 2017; 296: 855-67.

5. Taioli E, Bradlow HL, Garbers SV, et al. Role of estradiol metabolism and CYP1A1 polymorphisms in breast cancer risk. Cancer Detect Prev 1999; 23: 232-7.

6. Yang Q, Mas A, Diamond MP, Al-Hendy A. The mechanism and function of epigenetics in uterine leiomyoma development. Reprod Sci 2016; 23: 163-75.

7. Chen I, Motan T, Kiddoo D. Gonadotropin-releasing hormone agonist in laparoscopic myomectomy: systematic review and meta-analysis of randomized controlled trials. J Minim Invasive Gynecol 2011; 18: 303-9.

8. Steinauer J, Pritts EA, Jackson R, et al. Systematic review of mifepristone for the treatment of uterine leiomyomata. Obstet Gynecol 2004; 103: 1331-6.

9. Moroni RM, Martins WP, Ferriani RA, et al. Add-back therapy with GnRH analogues for uterine fibroids. Cochrane Database Syst Rev 2015; 20: CD010854.

10. Barnard EP, AbdElmagied AM, Laughlin-Tommaso SK, et al. Periprocedural outcomes comparing fibroid embolization and focused ultrasound: a randomized controlled trial and comprehensive cohort analysis. Am J Obstet Gynecol 2017; 216: 500. e1-11.

11. Haar G, Coussios C. High intensity focused ultrasound: physical principles and devices. Int J Hyperthermia 2007; 23: 89-104.

12. Coussios CC, Farny CH, Haar GT, et al. Role of acoustic cavitation in the delivery and monitoring of cancer treatment by high-intensity focused ultrasound (HIFU). Int I Hyperthermia 2007; 23: 105-20.

13. Hynynen K, Freund WR, Cline HE, et al. A clinical, non-invasive, MR imaging-monitored ultrasound surgery method. Radiographics 1996; 16: 185-95.

14. Kim YS. Advances in MR image-guided high-intensity focused ultrasound therapy. Int I Hyperthermia 2015; 31: 225-32.

15. He M, Jacobson H, Zhang C, et al. A retrospective study of ultrasound-guided high intensity focussed ultrasound ablation for multiple uterine fibroids in South Africa. Int J Hyperthermia 2018; 34: 1304-10.

16. Okada A, Morita Y, Fukunishi H, et al. Non-invasive magnetic resonance-guided focused ultrasound treatment of uterine fibroids in a large Japanese population: impact of the learning curve on patient outcome. Ultrasound Obstet Gynecol 2009; 34: 579-83.

17. Funaki K, Fukunishi H, Funaki T, et al. Magnetic resonance-guided focused ultrasound surgery for uterine fibroids: relationship between the therapeutic effects and signal intensity of pre-ex- 
isting T2-weighted magnetic resonance images. Obstet Gynecol 2007; 196: 184.e1-6

18. Keserci B, Duc NM. Magnetic resonance imaging parameters in predicting the treatment outcome of high-intensity focused ultrasound ablation of uterine fibroids with an immediate non-perfused volume ratio of at least $90 \%$. Acad Radiol 2018; 25: 1257-69.

19. Chen R, Kerserci B, Bi H, et al. The safety and effectiveness of volumetric magnetic resonance-guided high-intensity focused ultrasound treatment of symptomatic uterine fibroids: early clinical experience in China. J Ther Ultrasound 2016; 4: 27.

20. Tung SL, Chou TY, Tseng HS, Lee CM. A retrospective study of magnetic resonance-guided focused ultrasound ablation for uterine myoma in Taiwan. Taiwanese J Obstet Gynecol 2016; 55: 646-9.

21. Jeong JH, Hong GP, Kim YR, et al. Clinical consideration of treatment to ablate uterine fibroids with magnetic resonance imaging-guided high intensity focused ultrasound (MRgFUS): sonalleve. J Menopausal Med 2016; 22: 94-107.

22. Xu Y, Fu Z, Yang L, et al. Feasibility, safety, and efficacy of accurate uterine fibroid ablation using magnetic resonance imaging-guided high intensity focused ultrasound with shot sonication. J Ultrasound Med 2015; 34: 2293-303.

23. Mindjuk I, Trumm CG, Herzog P, et al. MRI predictors of clinical success in MR-guided focused ultrasound (MRgFUS) treatments of uterine fibroids: results from a single centre. Eur Radiol 2013; 25: 1317-28.

24. Tan N, Lu D, Raman S, Bahrami S. MR guided focused US for treatment of uterine fibroids: symptom reduction in a multicenter trial using a novel treatment algorithm. J Ther Ultrasound 2015; 3 (Suppl. 1): 91.

25. Jacoby VL, Kohi MP, Poder L, et al. PROMISe trial: a pilot, randomized, placebo-controlled trial of magnetic resonance-guided focused ultrasound for uterine fibroids. Fertil Steril 2016; 105: 773-80.

26. Park MJ, Kim YS, Rhim H, Lim HK. Safety and therapeutic efficacy of complete or near-complete ablation of symptomatic uterine fibroid tumors by MR imaging-guided high-intensity focused US therapy. J Vasc Interv Radiol 2014; 25: 231-9.

27. Himabindu Y, Sriharibabu M, Nyapathy V, Mishra A. Early evaluation of magnetic resonance imaging-guided focused ultrasound sonication in the treatment of uterine fibroids. Indian J Med Res 2014; 139: 267-72.

28. Gorny KRP, Borah BJP, Brown DLM, et al. Incidence of additional treatments in women treated with MR Guided focused US for symptomatic uterine fibroids: review of 138 patients with an average follow-up of 2.8 years. J Vasc Interv Radiol 2014; 25: 1506-12.

29. Funaki KK, Fukunishi H, Sawada K. Clinical outcomes of magnetic resonance-guided focused ultrasound surgery for uterine myomas: 24-month follow-up. Ultrasound Obstet Gynecol 2009; 34: 584-9.

30. Morita $\mathrm{Y}$, Ito $\mathrm{N}$, Hikida $\mathrm{H}$, et al. Non-invasive magnetic resonance imaging-guided focused ultrasound treatment for uterine fibroids - early experience. Eur J Obstet Gynecol Reprod Biol 2008; 139: 199-203.

31. Babashov V, Palimaka S, Blackhouse G, O'Reilly D. Magnetic resonance-guided high-intensity focused ultrasound (MRgHIFU) for treatment of symptomatic uterine fibroids: an economic analysis. Ont Health Technol Assess Ser 2015; 15: 1-61.

32. O 'Sullivan AK, Thompson D, Chu P, et al. Cost-effectiveness of magnetic resonance-guided focused ultrasound for the treatment of uterine fibroids. Int I Technol Assess Health Care 2009; 25: $14-25$.

33. Spies JB, Coyne K, Guaou Gulou N, et al. The UFS-QOL, a new disease-specific symptom and health-related quality of life questionnaire for leiomyomata. Obstet Gynecol 2002; 99: 290300.

34. Spies JB, Bradley LD, Guido R, et al. Outcomes from leiomyoma therapies: comparison with normal controls. Obstet Gynecol 2011; 117: 987-8

35. Manyonda IT, Bratby M, Horst JS, et al. Uterine artery embolization versus myomectomy: impact on quality of life - results of the FUME (Fibroids of the uterus: myomectomy versus embolization) trial. Cardiovasc Intervent Radiol 2012; 35 : 530-62.

36. Lee JS, Hong GY, Lee KH, Kim TE. Changes in anti-müllerian hormone levels as a biomarker for ovarian reserve after ultrasound-guided high-intensity focused ultrasound treatment of adenomyosis and uterine fibroid. BJOG 2017; 124: 18-22.

37. Cheung VY, Lam TP, Jenkins CR, et al. Ovarian reserve after ultrasound-guided high-intensity focused ultrasound for uterine fibroids: preliminary experience. J Obstet Gynaecol Can 2016; 38: 357-61.

38. Dobrotwir A, Pun E. Clinical 24 month experience of the first MRgFUS unit for treatment of uterine fibroids in Australia. J Med Imaging Radiat Oncol 2012; 56: 409-16.

39. Zowall H, Cairns JA, Brewer C, et al. Cost-effectiveness of magnetic resonance-guided focused ultrasound surgery for treatment of uterine fibroids. Obstet Gynecol Surv 2008; 63: 427-8.

40. Kong CY, Meng L, Omer ZB, et al. MRI-guided focused ultrasound surgery for uterine fibroid treatment: a cost-effectiveness analysis. Am J Roentgenol 2014; 203: 361-71.

41. Cain-Nielsen AH, Moriarty JP, Stewart EA, Borah BJ. Cost-effectiveness of uterine-preserving procedures for the treatment of uterine fibroid symptoms in the USA. J Comp Eff Res 2014; 3: 503-14.

42. Pron G. Magnetic resonance-guided high-intensity focused ultrasound (MRgHIFU) treatment of symptomatic uterine fibroids: an evidence-based analysis. Ont Health Technol Assess Ser 2015; 15: 1-86.

43. Peregrino PFM, de Lorenzo Messina M, Dos Santos Simoes R, et al. Review of magnetic resonance-guided focused ultrasound in the treatment of uterine fibroids. Clinics Sao Paulo 2017; 72: 637-41.

44. Laughlin-Tommaso SK, Weaver AL, Vaughan LE, et al. Long term outcomes in a randomized controlled trial of uterine artery embolization and MR-guided focused ultrasound: the first study. Fertil Steril 2017; 108: e26.

45. Junyan Li J, Chen X, Hu X. High-intensity focused ultrasound for treatment of recurrent uterine leiomyosarcoma: a case report and literature review. J Intern Med Res 2020; 48: 300060520942107.

46. Xu Y, Fu Z, Yang L, et al. Feasibility, safety, and efficacy of accurate uterine fibroid ablation using magnetic resonance imag- 
ing-guided high-intensity focused ultrasound with shot sonication. J Ultrasound Med 2015; 34: 2293-303.

47. Kok HP, Cressman E, Ceelen W, et al. Heating technology for malignant tumors: a review. Int I Hyperthermia 2020; 37: 71141.

Received: 26.04.2021, accepted: 4.07.2021. 